\title{
Accuracy of spectroscopy-based radioactive dating of stars
}

\author{
H.-G. Ludwig ${ }^{1,2}$, E. Caffau ${ }^{2}$, M. Steffen ${ }^{3}$, P. Bonifacio ${ }^{1,2,4}$, and L. Sbordone ${ }^{1,2}$ \\ 1 CIFIST Marie Curie Excellence Team, France \\ e-mail: Hans . Ludwig@obspm. fr \\ 2 GEPI, Observatoire de Paris, CNRS, Université Paris Diderot, Place Jules Janssen, 92190 Meudon, France \\ 3 Astrophysikalisches Institut Potsdam, An der Sternwarte 16, 14482 Potsdam, Germany \\ 4 Istituto Nazionale di Astrofisica, Osservatorio Astronomico di Trieste, via Tiepolo 11, 34143 Trieste, Italy
}

Received 10 August 2009 / Accepted 7 October 2009

\section{ABSTRACT}

\begin{abstract}
Context. Combined spectroscopic abundance analyses of stable and radioactive elements can be applied for deriving stellar ages. The achievable precision depends on factors related to spectroscopy, nucleosynthesis, and chemical evolution.

Aims. We quantify the uncertainties arising from the spectroscopic analysis, and compare these to the other error sources.

Methods. We derive formulae for the age uncertainties arising from the spectroscopic abundance analysis, and apply them to spectroscopic and nucleosynthetic data compiled from the literature for the Sun and metal-poor stars.

Results. We obtained ready-to-use analytic formulae of the age uncertainty for the cases of stable+unstable and unstable+unstable chronometer pairs, and discuss the optimal relation between to-be-measured age and mean lifetime of a radioactive species. Application to the literature data indicates that, for a single star, the achievable spectroscopic accuracy is limited to about $\pm 20 \%$ for the foreseeable future. At present, theoretical uncertainties in nucleosynthesis and chemical evolution models form the precision bottleneck. For stellar clusters, isochrone fitting provides a higher accuracy than radioactive dating, but radioactive dating becomes competitive when applied to many cluster members simultaneously, reducing the statistical errors by a factor $\sqrt{N}$.

Conclusions. Spectroscopy-based radioactive stellar dating would benefit from improvements in the theoretical understanding of nucleosynthesis and chemical evolution. Its application to clusters can provide strong constraints for nucleosynthetic models.
\end{abstract}

Key words. Sun: abundances - stars: abundances - stars: fundamental parameters - line: formation

\section{Introduction}

The determination of absolute ages for stars is of paramount importance in astrophysics since ages allow the timescales on which stellar populations and their chemical composition evolve to be established. The presence of long-lived unstable elements in stellar atmospheres offers a way to date stars by investigating their radioactive decay spectroscopically. In its simplest form, one determines the photospheric abundance of a pair of elements, of which one is unstable. By means of theoretical modeling of the nucleosynthetic production channels for the two elements, the original abundance of the unstable nucleus can be estimated from the abundance of the stable one. This allows the time-span over which the unstable element decayed to be determined.

The idea emerged early after the discovery of the rapid neutron capture process (short $r$-process) as the source of production of radionuclides such as thorium and uranium (see Cowan et al. 1991, and references therein for an account of its early applications). Since then it has been applied in a number of cases (among the most recent Frebel et al. 2007) but, typically, as a "side product" of analyses of the more general n-capture enrichment patterns. To our knowledge, the only effort in recent years to exploit radioactive dating to systematically derive the age of a significant sample of stars has been the one by del Peloso et al. (2005a,b,c).

One interest in such dating techniques lies in its applicability to field stars for which no distance information, hence no accurate information on surface gravity or absolute luminosity, is available, so that they cannot be placed with confidence on theoretical isochrones in the Hertzsprung-Russell diagram (HRD). For cluster stellar populations, on the other hand, HRD fitting usually provides rather precise absolute ages (e.g. Vandenberg et al. 1996). However, for stellar clusters (especially globular clusters), radioactive dating could produce an age estimate of comparable or even higher precision if applied to a large enough sample of stars, and would as such be very valuable as a test of the theoretical stellar isochrones.

Although potentially effective, spectroscopy-based radioactive dating is difficult to apply. The most easily measured longlived radioactive element, thorium, is only accessible (with the exception of strongly $r$-process enriched stars) through a single, weak line in the optical spectrum of stars, in which high accuracy is needed in the measurement to provide a reliable age. Besides lying on the red wing of a Fe-Ni blend, this unique Th II line is itself also blended by weaker lines of vanadium and cobalt. The relative strength of the various components for the Sun are discussed in Caffau et al. (2008) where the line-list of del Peloso et al. (2005a) was applied. Although rather easily measured, the most frequently used reference element, europium, is believed to constitute a less-than-optimal reference. In fact, its nucleosynthetic ties with Th are rather weak, and the production ratio is sensitive to the originating supernova characteristics (Kratz et al. 2007; Farouqi et al. 2008).

Besides the astrophysical problem that the $r$-process site has not been unambiguously identified, it is unclear how universal the resulting abundance pattern of the $r$-process nucleosynthesis actually is. In view of the complex nuclear reactions shaping the 
$r$-process it is not obvious that the outcome is the same for a potentially wide range of the physical conditions governing the formation site(s) (Goriely \& Clerbaux 1999; Arnould et al. 2007; Sneden et al. 2008; Roederer et al. 2009). To minimize the influence of poorly constrained physical conditions at the formation site it is desirable to use a reference element with an atomic mass as similar as possible to thorium. This should reduce the uncertainties in the formation properties to the uncertainties related to the properties of the involved nuclei as such, and environmental factors should cancel out. As pointed out before by various authors (e.g., Goriely \& Clerbaux 1999) the Th/U pair is in this sense optimal, however, again difficult to apply since uranium is difficult to detect spectroscopically. Recently, Kratz et al. (2007) suggested the element hafnium as suitable reference element for thorium-based radioactive dating. Hafnium is closer in mass to thorium than europium, but more importantly, Kratz and collaborators showed by detailed modeling that the production ratio $\mathrm{Th} / \mathrm{Hf}$ is not strongly varying over a wide range of neutron fluxes during the $r$-process. Since hafnium is a spectroscopically accessible element this makes it an interesting candidate as reference element.

Observationally, the forthcoming introduction of extremely large telescopes (ELTs) is likely to make radioactive dating of stars a much more exploitable technique, since the high signalto-noise $(\mathrm{S} / \mathrm{N})$ ratio required is one of the main difficulties associated with such studies. In this perspective, a precise assessment of the uncertainties associated with such technique is currently lacking, and attempted here. We derive the formulae for the precision to which spectroscopy can provide age estimates. They are subsequently applied to abundance analyses of stars compiled from the literature. This paper is a "spin-off" of our work (Caffau et al. 2008) on the thorium and hafnium abundance in the Sun, and the Th/Hf chronometer pair often serves as an example in the following sections.

\section{Formulae related to radioactive dating}

\subsection{Age uncertainties}

Any sample of radioactive nuclei decays according to the exponential law:

$n_{X}(t)=n_{X 0} \exp (-t / \tau)$

where $n_{X 0}$ is the number of nuclei at $t=0$, and $n_{X}$ the number of remaining nuclei at time $t$; $\tau$ is the mean lifetime. Explicitly, $t$ is given by

$t=\tau \ln \frac{n_{X 0}}{n_{X}}$.

Knowing the initial and present number of nuclei, one can obtain the time interval that has elapsed since $t=0$. In practice, to use the decay processes to determine an age, one measures the number of nuclei of the unstable species $\left(n_{X}\right)$ relative to the number of nuclei of a stable element which is related to $n_{X 0}$. This is, e.g., the case for the Th/Hf pair. Let us call $n_{R}$ the number of nuclei of the stable reference species related to $n_{X 0}$. Let us further assume that the two nuclei have a common nucleosynthetic origin and that the relation between the initial number of nuclei $n_{X 0}$ and $n_{R}$ is given by

$n_{X 0}=\beta n_{R}$

where $\beta$ is the production ratio between the respective numbers of nuclei. The production ratio may be predicted by theoretical considerations (see Kratz et al. 2007, and references therein). From Eqs. (2) and (3) one can write

$t=\tau \ln \frac{\beta n_{R}}{n_{X}}$

All astrophysically relevant unstable elements are trace species. Let us therefore suppose that the lines corresponding to the transitions we are interested in, are weak. In this case, the relation between equivalent width ( $W$ hereafter) and abundance $(n)$ is linear:

$W=C n \quad$ or $\quad \log W=\log C+\log n$,

$C$ being a constant defined by the line transition under consideration (dependent on oscillator strength and excitation potential) and the thermal structure of the stellar atmosphere under investigation.

Many lines - including the Th II resonance line - are blended with other species, and in practice the abundance is obtained by fitting a synthetic profile. In this case the equivalent width is obtained theoretically from the best-fitting profile and the underlying abundances. The error on the equivalent width may then be obtained by propagating the error in Eq. (5). One may also try to derive an equivalent width for the blended line by subtracting the theoretical equivalent width of the blending components from the total $W$ of the blend. However, in general, this ignores saturation effects, and thus the "corrected" $W$ differs from the true one. As an example we may consider the Th II resonance line in the Sun. Let us consider the theoretical computation of Caffau et al. (2008) based on a 3D CO ${ }^{5}$ BOLD model: the $W$ of the blend Th II $+\mathrm{Co}$ I $+\mathrm{V}$ II, for an assumed abundance $A(\mathrm{Th})=0.09$ is $0.74 \mathrm{pm}$, the $W$ of just the Co I+V II blend is $0.31 \mathrm{pm}$, while $W(\mathrm{Th})=0.46 \mathrm{pm}$. In spite of the fact that all the lines involved are very weak, it is clear that the "corrected" $W(0.43 \mathrm{pm})$ differs from $W(\mathrm{Th})$, due to saturation effects. Thus one should always use the equivalent width of the line, rather than the "corrected" $\mathrm{W}$, although in many cases the difference is so small that it may be safely ignored. Alternatively, one may work with Eq. (9) and use the obtained abundances directly. In this case one has to establish uncertainties by Monte-Carlo simulations.

Substituting Eqs. (3) and (5) into Eq. (2) we obtain

$$
\begin{aligned}
t & =\tau \ln \left(\beta \frac{W_{R}}{W_{X}} \frac{C_{X}}{C_{R}}\right) \\
& =\tau\left\{\ln \beta+\ln W_{R}-\ln W_{X}+\ln \left(\frac{C_{X}}{C_{R}}\right)\right\} .
\end{aligned}
$$

The achievable precision in $t$ is hence limited by the uncertainty in the knowledge of $\beta$, errors in the measurement of $W_{X}$ and $W_{R}$, as well as by systematic errors in modeling the stellar atmosphere and line transitions, i.e. errors of the constants $C_{X}$ and $C_{R}$.

From the standard formula for the propagation of uncorrelated errors we get for the variance of $t$

$$
\begin{aligned}
\sigma(t)= & \tau\left\{\left(\frac{\sigma(\beta)}{\beta}\right)^{2}+\left(\frac{\sigma\left(W_{R}\right)}{W_{R}}\right)^{2}+\left(\frac{\sigma\left(W_{X}\right)}{W_{X}}\right)^{2}\right. \\
& \left.+\left(\frac{\sigma\left(C_{X} / C_{R}\right)}{C_{X} / C_{R}}\right)^{2}\right\}^{1 / 2} .
\end{aligned}
$$

This equation is valid under the presumption that the errors of $\beta$, $W_{X}, W_{R}$, and $\left(C_{X} / C_{R}\right)$ are uncorrelated. The errors of $C_{X}$ and $C_{R}$ themselves may often be more or less correlated. In fact, Th and 
Hf abundances are derived from singly ionized species which constitute the dominant ionization stage in the late-type stars of interest here. Moreover, the diagnostic lines of Th and $\mathrm{Hf}$ emerge from low lying energy levels which makes the Boltzmann factor and consequently the temperature sensitivity of the level populations modest. The similar atomic properties of Th and $\mathrm{Hf}$ lead to similar formation regions in the photosphere. Hence, all systematic errors due to uncertainties in gravity, effective temperature and even the details of the thermal structure of the employed model atmosphere should largely cancel out. Hf is the heaviest element prior to Th whose observable spectroscopic signatures are this favorable. What is left is the systematic error related to the $g f$-values.

Taking the Sun and the Th/Hf pair as an example, we have $\sigma\left(W_{R}\right) / W_{R} \approx 0.03 / 0.3=0.1$ (see Hf II $\lambda 409.3 \mathrm{~nm}$ in Table 3 in Caffau et al. 2008), and $\sigma\left(W_{X}\right) / W_{X} \approx 0.03 / 0.4=0.075$ (see Table 4 in Caffau et al. 2008). Since ${ }^{232} \mathrm{Th}$ has a mean lifetime of $\tau=20.3 \mathrm{Gyr}$, we obtain from Eq. (7) under the most optimistic conditions, assuming $\sigma(\beta)=0$ and $\sigma\left(C_{X} / C_{R}\right)=0$, an error in the age determination of the order of $\sigma(t) \approx 2.5 \mathrm{Gyr}$.

Even this rather limited precision is probably beyond the present state-of-the-art in stellar spectroscopy. However it is not implausible to reach a precision of the order of $2 \mathrm{Gyr}$ in the future. The uncertainties due to the $\mathrm{S} / \mathrm{N}$ in the spectrum may be considerably reduced by the next generation of ELTs, and uncertainties in the blending lines can be reduced through laboratory measurements and/or theoretical calculations. The precision currently attainable on the dating of Globular Clusters, using Main Sequence fitting, is a factor of two better (about $1 \mathrm{Gyr}$ ), however it may not be applied to field stars. For field stars which are evolved off the Main Sequence, if the distance is known, an age can be derived through comparison with theoretical isochrones. However, distances are difficult to measure and a small error in the distance propagates to a great error in age. In principle, radioactive dating has the potential to provide ages of individual field stars, even if their distances are not precisely known.

Unfortunately, in the literature equivalent widths and their uncertainties are usually not given so that a direct application of the previous formulae is not possible. In Sect. 2.2 we will see that they are nevertheless useful to derive a statement of the optimal decay time of a species for measuring a particular time interval. For now, we proceed by listing the previous relations in terms of the logarithmic abundances $A(X)=\log \epsilon_{X} \equiv \log n_{X} / n_{H}+12$. Equation (4) for the age becomes

$t=\tau \ln 10\{\log \beta+A(R)-A(X)\}$,

with the corresponding uncertainty

$$
\begin{aligned}
\sigma(t)= & \tau \ln 10\left\{\sigma^{2}[\log \beta]+\sigma^{2}[A(X)]+\sigma^{2}[A(R)]\right. \\
& -2 \sigma[A(X)] \sigma[A(R)] \operatorname{Cor}[A(X), A(R)]\}^{1 / 2} .
\end{aligned}
$$

Cor [.,.] denotes the linear correlation coefficient. In deriving Eq. (9), it was assumed that $\beta$ is uncorrelated with $A(X)$ and $A(R)$. Relation (9) is consistent with Eq. (7) as long as the statistical bias due to the change from linear to logarithmic quantities is small, i.e. that for any involved quantity $q$

$\frac{\sigma(q)}{q} \approx \sigma(\ln q)=\ln 10 \sigma(\log q)$.

Equation (9) now explicitly contains the correlation among the measurements of the abundances of the two involved species. If abundance errors are positively correlated - as discussed previously and the Th/Hf pair serving as example - this can lead to a substantial reduction of the overall error with respect to the uncorrelated case. However, care has to be exercised when applying the formulae to literature data (as we intend to do). Quoted abundance errors are often purely statistical, uncorrelated uncertainties while correlated uncertainties related to atmospheric models are specified separately by providing sensitivities against changes of the stellar parameters.

\subsection{Optimal decay times}

We now discuss how the achievable precision depends on the time interval $t$ we want to measure and the decay time of the radioactive species, $\tau$. From Eq. (7) or (9) it might appear that a radioactive species with short mean lifetime $\tau$ provides the highest precision on $t$. However, this reasoning ignores the fact that a rapid decay $(\tau \ll t)$ makes it more difficult to measure $W_{X}$ precisely. To make this connection manifest we eliminate $W_{X}$ and rewrite Eq. (7) as

$$
\begin{aligned}
\frac{\sigma(t)}{t}= & \frac{\tau}{t}\left\{\left(\frac{\sigma\left(W_{R}\right)}{W_{R}}\right)^{2}\left(1+\left(\frac{C_{R}}{\beta C_{X}}\right)^{2} \exp (2 t / \tau)\right)\right. \\
& \left.+\left(\frac{\sigma(\beta)}{\beta}\right)^{2}+\left(\frac{\sigma\left(C_{X} / C_{R}\right)}{C_{X} / C_{R}}\right)^{2}\right\}
\end{aligned}
$$

where we have used the relations

$\frac{W_{R}}{W_{X}}=\frac{C_{R}}{\beta C_{X}} \exp (t / \tau)$

and

$\sigma\left(W_{R}\right)=\sigma\left(W_{X}\right)$

Equation (12) is obtained by combining Eqs. (1), (3), and (5), while Eq. (13) is a consequence of Cayrel's formula (Cayrel 1988) which, for a spectrum that is photon noise dominated, states that the uncertainty of the $W$ measurement $\sigma(W)$ depends on the FWHM of the line, the pixel size, and the $\mathrm{S} / \mathrm{N}$ of the spectrum. For spectra of moderate resolution $(R \leq 80000)$, the FWHM is essentially the same for all lines since they are not fully resolved and the FWHM is simply set by the spectrograph's resolution. This is also true for higher resolution spectra if the atoms under consideration do not have a very large difference in mass, and therefore thermal broadening, or if the dominant broadening is non-thermal. In the case of the $\mathrm{Th} / \mathrm{Hf}$ pair, the mass difference is not large, and in late-type stars the macroturbulent plus rotational broadening is often of the same order of magnitude or greater than the thermal broadening. We may therefore assume that $\sigma(W)$ is the same for any line in a given spectrum. Assuming again $\sigma(\beta)=0$, and $\sigma\left(C_{X} / C_{R}\right)=0$, and noting that $\sigma\left(W_{R}\right) / W_{R}$ is time independent, the relative error of $t$ becomes

$$
\begin{aligned}
\frac{\sigma(t)}{t} & =\frac{\sigma\left(W_{R}\right)}{W_{R}} \frac{\tau}{t}\left(1+\left(\frac{C_{R}}{\beta C_{X}}\right)^{2} \exp (2 t / \tau)\right)^{1 / 2} \\
& \equiv \frac{\sigma\left(W_{R}\right)}{W_{R}} F\left(\frac{t}{\tau} ; p\right) \text { with } p \equiv \frac{C_{R}}{\beta C_{X}} .
\end{aligned}
$$

For brevity, we introduced an auxiliary function $F$ of argument $\frac{t}{\tau}$ and parameter $p$ in Eq. (14). The smaller $F\left(\frac{t}{\tau}\right)$ at given $p$, the higher the precision of the age determination. The best (relative) accuracy is obtained for the time where the function $F$ shows a minimum. As shown in Fig. 1, the function has a minimum 


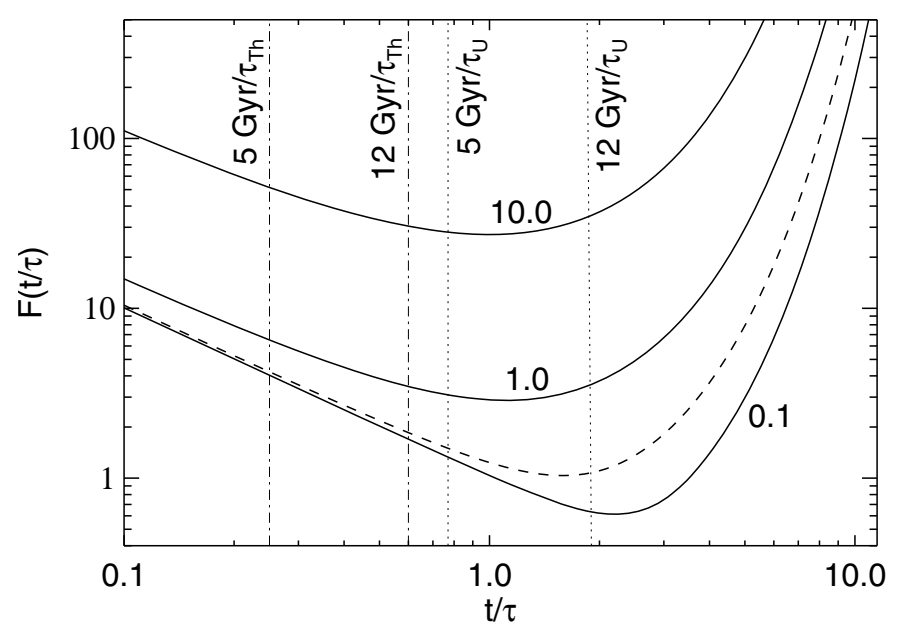

Fig. 1. The function $F\left(\frac{t}{\tau} ; p\right)$ defined in Eq. (14) plotted as a function of $\frac{t}{\tau}$ for different parameters $p$ (solid curves). The dashed line shows the case of the $\mathrm{Th} / \mathrm{Hf}$ chronometer pair under solar conditions (Th II $\lambda 401.8 \mathrm{~nm}$ and Hf II $\lambda 409.3 \mathrm{~nm}$ line pair, $p=0.26)$. The the dotted and dash-dotted vertical lines mark two characteristic ages, 5 and $12 \mathrm{Gyr}$, taking the Th $(20.3 \mathrm{Gyr})$ and $\mathrm{U}(6.5 \mathrm{Gyr})$ mean lifetimes as reference.

near $t \approx \tau$. Left of the minimum $(t<\tau), F$ decreases slowly with time, while it increases more steeply beyond the minimum $(t>\tau)$. We can conclude that an optimal relative precision for radioactive dating is obtained when one measures a time comparable to the mean lifetime of the unstable element - a perhaps intuitively expected result, put on a quantitative footing here. Of course, our analysis is idealized by assuming a single, isolated line for both the stable and decaying species. In practice, often several lines are considered and line strengths are measured via spectral synthesis in order to account for line blending. However, we think that the overall picture in this more complex situation does not change qualitatively.

We finally consider radioactive dating when measuring two lines from two transitions of two unstable elements which we call $X$ and $Y$. There are two decay laws to be considered for the two elements:

$n_{X}=n_{X 0} \exp \left(-t / \tau_{X}\right) \quad$ and $\quad n_{Y}=n_{Y 0} \exp \left(-t / \tau_{Y}\right)$.

We suppose that $\tau_{X}<\tau_{Y}$, and that the two elements are, at least partially, formed in the same nucleosynthetic process, so that the abundances of nuclei of the two elements at the initial instant of time are related by $n_{X 0}=\beta n_{Y 0}$. In this way, we obtain

$n_{X}=\beta n_{Y} \exp (-t / \tau)$,

once $\tau$ is defined as

$\frac{1}{\tau}=\frac{1}{\tau_{X}}-\frac{1}{\tau_{Y}}$

We then find the same result for $\sigma(t) / t$ as obtained above in Eq. (14), except that $\tau$ now depends on the mean lifetimes of the two elements considered according to Eq. (17), and the reference element $R$ is identified with the longer lived species $Y$ at its present abundance.

In practice, the only two radioactive nuclei which have been used to date stars are ${ }^{232} \mathrm{Th}$ and ${ }^{238} \mathrm{U}$ with mean lifetimes of $20.3 \mathrm{Gyr}$ and $6.5 \mathrm{Gyr}$, respectively. From Eq. (17) the effective $\tau$ for the Th/U pair amounts to 9.6 Gyr. From Fig. 1 it is clear that the Th/U pair as well as U/R pairs ( $R$ for any stable $r$-process element) are well suited for measuring great ages around $12 \mathrm{Gyr}$, with $\mathrm{Th} / \mathrm{R}$ being competitive depending on the actual value of the $p$ parameter. Towards ages of a few Gyr the sensitivity of $\mathrm{U} / \mathrm{R}$ pairs is about 2 to 3 times higher than the one of Th/R pairs, again depending on $p$. We repeat that only the decay times enter the considerations here; it is usually more difficult to measure $\mathrm{U}$ than Th abundances so that the advantage of the shorter life time of $U$ is offset. Finally, we ask whether there are other suitable naturally occurring radioactive elements with lifetimes even more favorable for measuring ages in the range of a few Gyr. The answer is "no": the lifetimes are either much too short or much too long - besides further complications like exceedingly low abundances of the respective isotopes or the availability of suitable spectral lines.

\section{Ages of metal-poor stars}

As an application of the formulae we derived for the errors on age, we consider metal-poor stars for which the thorium abundance has been measured. For all these stars the abundance of at least one other $r$-process element is available (see Table A.1). Using various production ratios $\beta$ available in the literature (see Table A.2), we computed the ages of these stars using Eq. (8) and their uncertainties with Eq. (9). Correlations were neglected; correlations due to uncertainties in stellar parameters are usually not included in the error budget, and correlations emerging from the data reduction (e.g. background reduction) not specified by the authors. Our results are summarized in Table 1. For star HE 1523-0901 the abundances of the single elements were not available, but in Frebel et al. (2007) abundance ratios are reported, and measurement errors on the individual abundances which is sufficient for our purposes. By using different production ratios we intended to separate the systematic uncertainties related to nucleosynthesis and chemical evolution from the spectroscopic uncertainties as such. Since the production ratios are treated separately here their related uncertainty in the evaluation of $\sigma(t)$ with Eq. (9) is set to zero, $\sigma^{2}[\log \beta]=0$.

We like to point out that we applied the spectroscopy-related uncertainties of the abundances as given by the authors. There is no general consensus about the appropriate way to estimate the error on abundances, and the approach varies from author to author. For those elements which have several measurable lines the error can be estimated by looking at the rms line-toline abundance scatter. One further might divide this error by $\sqrt{N}$ where $N$ is the number of lines, provided the errors on each line are independent. However, this assumption is often questionable, since in most cases the lines are measured from the same spectrum. Any error which affects the whole spectrum (e.g. an error in the background subtraction) will affect all the lines in the same (or at least in a similar) way. We thus argue that it is more realistic and conservative not to invoke this factor $1 / \sqrt{N}$.

The ages of the individual metal-poor stars in Table 1 show a great spread when considering different $r$-production ratios, $\beta$, and chronometer pairs. Also the age uncertainties stemming from the spectroscopically determined abundances, $\sigma_{\text {Age }}$, are substantial. We note that our spectroscopy-related uncertainties are commonly greater than the estimates given by the authors themselves. Figures 2 and 3 provide a graphical representation of the situation for the stars CS 22892-052 and CS 31082001, respectively. We picked these objects since spectroscopic measurements for a greater number of chronometer pairs exist. CS 31082-001 is of particular interest since it is strongly $r$-process element enhanced allowing the measurement of the Th abundance exceptionally from several lines making it more 
H.-G. Ludwig et al.: Accuracy of spectroscopy-based radioactive stellar dating

Table 1. Ages and age uncertainties $\sigma_{\text {Age }}$ of metal-poor stars and the Sun obtained for different chronometer pairs $X / Y$.

\begin{tabular}{|c|c|c|c|c|c|c|}
\hline$X / Y$ & Object & \multicolumn{4}{|c|}{$\begin{array}{l}\mathrm{Age}^{a} \\
{[\mathrm{Gyr}]}\end{array}$} & $\begin{array}{r}\sigma_{\mathrm{Age}} \\
{[\mathrm{Gyr}]}\end{array}$ \\
\hline $\mathrm{Th} / \mathrm{Eu}$ & HD 115444 & 11.7 & 10.4 & 12.3 & 14.6 & 6.9 \\
\hline $\mathrm{Th} / \mathrm{Os}$ & HD 115444 & 30.1 & 24.3 & & & 7.7 \\
\hline $\mathrm{Th} / \mathrm{Eu}$ & CS $22892-052^{b}$ & 14.8 & 13.4 & 15.4 & 17.6 & 6.4 \\
\hline $\mathrm{Th} / \mathrm{Hf}$ & CS 22892-052 & 27.7 & & & & 7.4 \\
\hline $\mathrm{Th} / \mathrm{Os}$ & CS 22892-052 & 19.6 & 14.8 & & & 18.5 \\
\hline $\mathrm{Th} / \mathrm{Eu}$ & CS 22892-052 & 15.4 & 13.1 & 15.0 & 17.3 & 5.3 \\
\hline Th/Os & CS 22892-052 & 24.4 & 18.6 & & & 5.1 \\
\hline $\mathrm{Th} / \mathrm{Ir}$ & CS 22892-052 & 25.8 & 19.1 & 25.2 & & 12.9 \\
\hline $\mathrm{Th} / \mathrm{Eu}$ & CS 22892-052 & 12.1 & 11.7 & 13.7 & 15.9 & 4.8 \\
\hline $\mathrm{Th} / \mathrm{Hf}$ & CS 22892-052 & 24.6 & & & & 6.1 \\
\hline $\mathrm{Th} / \mathrm{Os}$ & CS 22892-052 & 26.0 & 20.3 & & & 7.7 \\
\hline $\mathrm{Th} / \mathrm{Ir}$ & CS 22892-052 & 19.0 & 13.3 & 19.4 & & 6.1 \\
\hline $\mathrm{Th} / \mathrm{Eu}$ & $\mathrm{BD}+173248$ & 7.6 & 6.3 & 8.3 & 10.5 & 5.3 \\
\hline Th/Os & $\mathrm{BD}+173248$ & 27.7 & 22.0 & & & 6.1 \\
\hline $\mathrm{Th} / \mathrm{Ir}$ & $\mathrm{BD}+173248$ & 20.7 & 14.0 & 19.1 & & 7.7 \\
\hline $\mathrm{Th} / \mathrm{Eu}$ & HD 221170 & 11.7 & 10.4 & 12.3 & 14.6 & 4.2 \\
\hline $\mathrm{Th} / \mathrm{Hf}$ & HD 221170 & 21.9 & & & & 2.9 \\
\hline $\mathrm{Th} / \mathrm{Os}$ & HD 221170 & 27.1 & 22.3 & & & 5.3 \\
\hline $\mathrm{Th} / \mathrm{Ir}$ & HD 221170 & 20.7 & 14.0 & 19.1 & & 6.1 \\
\hline $\mathrm{Th} / \mathrm{Eu}$ & CS 31082-001 & 0.9 & -1.5 & 0.5 & 2.7 & 2.9 \\
\hline $\mathrm{Th} / \mathrm{Hf}$ & CS 31082-001 & 15.2 & & & & 8.0 \\
\hline Th/Os & CS 31082-001 & 17.9 & 12.1 & & & 5.9 \\
\hline $\mathrm{Th} / \mathrm{Ir}$ & CS 31082-001 & 6.5 & -0.2 & 5.9 & & 4.1 \\
\hline $\mathrm{U} / \mathrm{Th}$ & CS 31082-001 & 16.6 & 13.3 & 14.6 & & 5.9 \\
\hline $\mathrm{Th} / \mathrm{Eu}$ & HE 1523-0901 & 11.4 & 9.5 & 11.0 & 13.2 & 3.1 \\
\hline Th/Os & HE 1523-0901 & 16.9 & 10.1 & & & 3.1 \\
\hline $\mathrm{Th} / \mathrm{Ir}$ & HE 1523-0901 & 18.0 & 12.3 & 17.4 & & 3.1 \\
\hline $\mathrm{U} / \mathrm{Th}$ & HE 1523-0901 & 15.0 & & & & 2.6 \\
\hline $\mathrm{Th} / \mathrm{Eu}$ & M51 K341 & 11.0 & 9.7 & 12.6 & 13.9 & 6.9 \\
\hline $\mathrm{Th} / \mathrm{Eu}$ & M51 K462 & 14.1 & 12.7 & 14.7 & 16.9 & 6.9 \\
\hline $\mathrm{Th} / \mathrm{Eu}$ & M51 K583 & 6.6 & 4.3 & 7.2 & 8.5 & 6.9 \\
\hline $\mathrm{Th} / \mathrm{Hf}$ & M4 L1411 & 32.5 & & & & 3.4 \\
\hline $\mathrm{Th} / \mathrm{Hf}$ & M4 L1411 & 32.5 & & & & 3.4 \\
\hline $\mathrm{Th} / \mathrm{Hf}$ & M4 L1501 & 25.5 & & & & 3.4 \\
\hline $\mathrm{Th} / \mathrm{Hf}$ & M4 L1514 & 37.2 & & & & 3.4 \\
\hline $\mathrm{Th} / \mathrm{Hf}$ & M4 L2406 & 32.5 & & & & 3.4 \\
\hline Th/Hf & M4 L2617 & 23.2 & & & & 3.4 \\
\hline $\mathrm{Th} / \mathrm{Hf}$ & M4 L3209 & 30.2 & & & & 3.4 \\
\hline Th/Hf & M4 L3413 & 23.2 & & & & 3.4 \\
\hline Th/Hf & M4 L4511 & 27.8 & & & & 3.4 \\
\hline Th/Hf & M4 L4611 & 37.2 & & & & 3.4 \\
\hline Th/Hf & M5 IV-81 & 18.5 & & & & 3.4 \\
\hline $\mathrm{Th} / \mathrm{Hf}$ & M5 IV-82 & 18.5 & & & & 3.4 \\
\hline $\mathrm{Th} / \mathrm{Eu}$ & HD 108577 & 7.8 & 6.2 & 8.4 & 10.1 & 3.4 \\
\hline $\mathrm{Th} / \mathrm{Eu}$ & HD 115444 & 9.6 & 8.1 & 10.3 & 11.9 & 3.6 \\
\hline $\mathrm{Th} / \mathrm{Eu}$ & HD 186478 & 16.6 & 15.1 & 17.3 & 18.9 & 4.3 \\
\hline $\mathrm{Th} / \mathrm{Eu}$ & $\mathrm{BD}+82856$ & 7.3 & 5.8 & 8.0 & 9.6 & 5.5 \\
\hline $\mathrm{Th} / \mathrm{Eu}$ & M92 VII-18 & 6.8 & 5.3 & 7.5 & 9.1 & 5.3 \\
\hline $\mathrm{Th} / \mathrm{Eu}$ & Sun & 3.3 & 1.7 & 3.9 & 5.6 & 2.0 \\
\hline Th/Hf & Sun & 22.3 & & & & 2.3 \\
\hline Th/Os & Sun & 14.1 & 8.6 & & & 4.9 \\
\hline $\mathrm{Th} / \mathrm{Ir}$ & Sun & 10.1 & 4.0 & 9.6 & & 4.9 \\
\hline
\end{tabular}

${ }^{a}$ Ages and age uncertainties are obtained from formulae (8) and (9) using the abundances listed in Table A.1. The four "Age" columns give ages based on production rations $\beta$ of Kratz et al. (2007), Sneden et al. (2003), Schatz et al. (2002), and Cowan et al. (2002) (in that order) according to Table A.2. The Solar ages are calculated applying $r$-process fractions as given in Table A.3.

${ }^{b}$ Three independent measurements were obtained for this star which is the reason why certain chronometer pair/object combinations are listed multiple times.

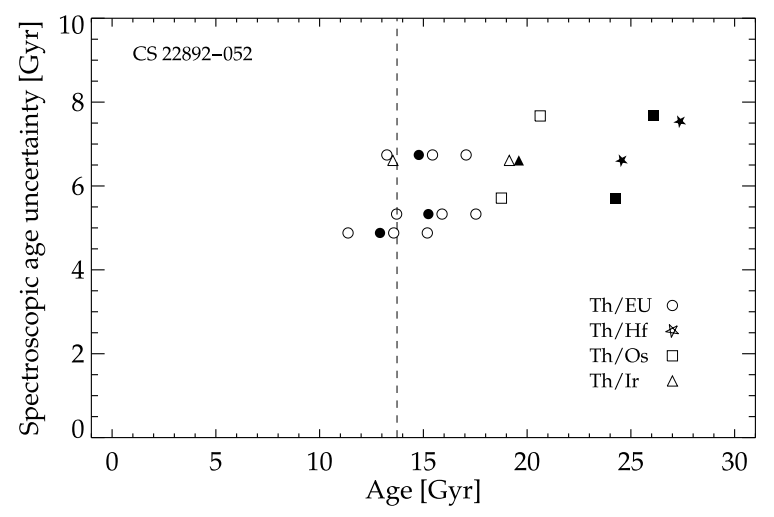

Fig. 2. Ages and spectroscopic age uncertainties for star CS 22892-052 determined from various chronometer pairs (symbols) assuming up to four different production ratios. Filled symbols refer to the production rations of Kratz et al. (2007). The dashed line indicates the age of the universe. Sneden et al. (2003) give a radiochronometric age estimate of $14.2 \pm 3$ Gyr for this star.

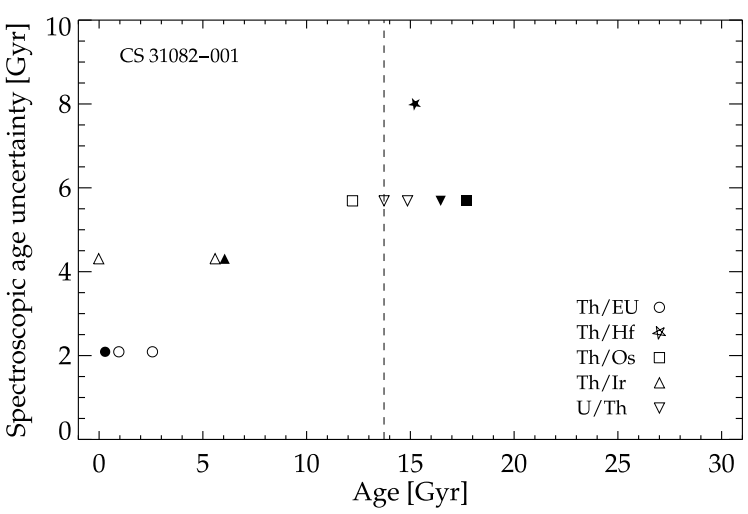

Fig. 3. Same as Fig. 2 for star CS 31082-001. In addition, for this star $\mathrm{U} / \mathrm{Th}$ ages are available. Hill et al. (2002) give a radiochronometric age estimate of $14.0 \pm 2.4 \mathrm{Gyr}$ for this stars.

reliable. For CS 22892-052 we left out the age estimates with the highest uncertainties, stemming from $\mathrm{Th} / \mathrm{Os}$ and $\mathrm{Th} / \mathrm{Ir}$, since they were later improved substantially. Both figures illustrate that the dispersion due to different production ratios is often greater than the dispersion due to abundance uncertainties. This is even the case if one restricts the comparison to production ratios originating from the same author. As already pointed out in the original paper on the abundance analysis of CS 31082-001 by Hill et al. (2002) the age derived from the Th/Eu pair is obviously unrealistic despite rather accurate abundance measurements. The same holds to lesser extend for the Th/Ir pair. This points to shortcomings in our understanding of the $r$-process production ratios and/or the chemical history of the star. For CS 22892-052 all the reference elements except Eu lead to exceedingly great ages. One might speculate that the $\mathrm{Th} / \mathrm{Hf}$ age might be enlarged by an s-process contribution to the $\mathrm{Hf}$ abundance: although the star has a very low metalicity, it is also carbon enhanced, so that one cannot rule out the possibility that it may have a (so far undetected) companion which went through the AGB phase, polluting the photosphere of the primary. However, even this hypothesis cannot be invoked to explain the great $\mathrm{Th} / \mathrm{Os}$ and $\mathrm{Th} / \mathrm{Ir}$ ages. Os and Ir are in fact supposed to show only minor $s$-process contributions (Arlandini et al. 1999). Again, we must conclude that large systematic uncertainties still exist in the theoretical calculations of production ratios $\beta$. 
At this point one may ask what accuracy has been reached in spectroscopically measured abundance ratios necessary for radioactive dating - in particular for metal-poor stars -, and what are the perspectives for improving these accuracies in the future. Both questions are not easy to answer. Uncertainties given by individual authors are often on the optimistic side. We think that a comparison among different authors provides a more robust measure of the actually present - as opposed to published - uncertainties. Such a comparison might still be biased towards too small error bars since different authors often draw from the same sources for oscillator strengths, or may use the same observational material. Hence, the analyses are not independent. Sneden et al. (2009) performed such a comparison of the abundances which they obtained for the metal-poor $r$-process enriched halo giant CS 31082-001 with studies of Hill et al. (2002) and Honda et al. (2004). We repeat this comparison here for the obtained abundance ratios. In the comparison Honda-Sneden the list of common elements is $\mathrm{La}, \mathrm{Ce}, \mathrm{Pr}, \mathrm{Nd}, \mathrm{Sm}, \mathrm{Eu}, \mathrm{Gd}, \mathrm{Tb}, \mathrm{Dy}, \mathrm{Er}$, $\mathrm{Tm}, \mathrm{Yb}$, in Hill-Sneden La, Ce, Pr, Nd, Sm, Eu, Gd, Tb, Dy, Er, Tm, Hf. Abundance ratios among these elements can be taken as a rather good model for the Th/Hf pair since their lines emerge from the first, dominant ionization stage, and from levels of low excitation potential (Lawler et al. 2009; Sneden et al. 2009) similar to the situation for the Th/Hf pair. Taking the abundance ratios from the individual studies ensures that correlation effects reducing the errors on abundance ratios are fully taken into account. For the rare earth elements often many lines are available for the determination of their abundance. This luxury is not afforded by Th or Hf so that we still have a likely bias towards too high accuracy when taking the rare earths as example.

From the lists of elements in common we formed all possible abundance ratios (except for an element with itself), and calculated the difference of the logarithmic ratios between the various studies. For the pair Hill-Sneden we find a dispersion among the differences of the logarithmic ratios of $0.14 \mathrm{dex}$, for Honda-Sneden 0.12 dex. In the case of Hill-Sneden, in particular the discrepant Hf abundances have to be noted. In the case of Honda-Sneden, a substantial part of the dispersion is driven by the elements Tm and Tb; leaving them out reduces the dispersion to 0.05 dex; however, this is not suggested by, e.g., excessive errors on their abundance as stated by the authors. We emphasize that at least in part the various groups use the same oscillator strengths. From this exercise we conclude that attributing an uncertainty of about $0.1 \mathrm{dex}$ to the $\mathrm{Th} / \mathrm{Hf}$ abundance ratio is perhaps conservative but not grossly over-estimating the actual value.

How to improve the accuracy? Sneden et al. (2009) report a line-to-line scatter of typically $0.07 \mathrm{dex}$ in a given star when determining abundances of a rare earth element and applying the high-quality oscillator strength of Lawler et al. (2009, and references therein). A sub-optimal choice of the model atmosphere introduces some scatter, but certainly errors in the oscillator strength are an important contributor. Since two abundances are involved in the determination of a ratio, an uncertainty of about 0.1 dex would result when an individual abundance is given to 0.07 dex accuracy. It is often argued that by averaging over abundances of $N$ individual lines one improves the obtained accuracy by a factor $\sqrt{N}$. However, this would only be the case if the scatter among the oscillator strength is of random nature which is far from obvious. So, even more accurate oscillator strengths of the involved line transitions are desirable. This also relates to lines of blending species which one has to deal with. At this point an appropriate theoretical description of the line formation process also comes into play which should account for departures from local thermodynamic equilibrium and gas-dynamical effects. All this is alleviated by having high signal-to-noise and high resolution spectra at hand which ELTs will be able to provide. One often has to work with spectra where a line profile is sampled with only very few points. Better sampling usually permits more accurate measurements. We are convinced that we are not stuck at the present level of accuracy to which abundance ratios used in radioactive dating can be determined but not insignificant effort is necessary to make substantial progress.

\section{The Solar age}

As another application of the dating formulae we tried to deduce the solar age. We start by taking the Th/Hf pair as an example since we worked recently ourselves on this chronometer pair (Caffau et al. 2008) having all relevant spectroscopic information at hand. In the case of the $401.9 \mathrm{~nm}$ Th II / $409.3 \mathrm{~nm}$ Hf II line pair, our favorite spectral synthesis code gives $C_{R} / C_{X}=0.13$. For the Sun one has to consider two complications which are less severe for metal-poor stars:

1. the Th and Hf observed in the solar system are not the result of a single $r$-process event, but rather of the superposition of many events;

2. while Th is produced only through the $r$-process, Hf is in part produced by the $s$-process.

To take into account the first point one should use an appropriate model of Galactic chemical evolution (see, e.g., del Peloso et al. 2005b). We ignore this aspect and assume a single $r$-process event, to see if at least a reasonable estimate of the solar age can be derived. The second point instead can easily be taken into account by using the solar $r$-process fraction of $\mathrm{Hf}$ nuclei. This has been estimated by Lawler et al. (2007) to be $56.6 \%$ of the total Hf nuclei. For the production ratio we assume $\beta=0.862$, from Table 2 of Kratz et al. (2007). The curve $F\left(\frac{t}{\tau} ; p=0.26\right)$ related to this case is depicted as a dashed line in Fig. 1. For ages 5 and 12 Gyr (i.e. for times corresponding to the age of the Sun and to the age of Galactic Globular Clusters, respectively), we find $\sigma(t) / t=4.3 \times \sigma\left(W_{R}\right) / W_{R}$ and $1.9 \times \sigma\left(W_{R}\right) / W_{R}$, respectively. We give these numbers mainly for illustrating the application of the formulae involving the equivalent widths. In the solar case they are not strictly applicable since when deriving Eq. (14) we assumed that uncertainties in the observed spectra are related to the photon noise. This is certainly not the case for Solar spectra. The uncertainties are related to the systematics, in particular blending of the thorium line.

Turning now to spectroscopically measured abundances, Table 1 lists the obtained ages and their uncertainties, again for different chronometer pairs and assumptions about production ratios and $r$-process fractions (see Tables A.1-A.3). The Th/Hf age, $22.3 \mathrm{Gyr}$, comes out to be greater than the age of the universe, $13.73 \mathrm{Gyr}$, as estimated from the fluctuations of the Cosmic Microwave Background (Spergel et al. 2007). By using the $A(\mathrm{Hf})=0.88$ value suggested by Lawler et al. (2007), only 0.01 dex higher than the abundance we use, the age comes out even a bit greater by $0.5 \mathrm{Gyr}$. Even if the solar abundance of $\mathrm{Hf}$ and Th were produced in a number of distinct supernova events at different times, the resulting mean age as computed from Eq. (8) cannot be greater than the time elapsed since the earliest supernova event, which cannot have occurred earlier than 13.73 Gyr ago. We have ignored the destruction of Th by photons in stellar interiors. According to del Peloso et al. (2005b) the matter cycled through stars may have a reduction of $10-20 \%$ in 
Th. However, even when increasing the Solar Th abundance by $20 \%$, one still derives an age of $18.2 \mathrm{Gyr}$ from Eq. (2). We have to conclude that either the assumed value of $\beta$, or the $r$-process fraction of $\mathrm{Hf}$ is incorrect. Among the other chronometer pairs $\mathrm{Th} / \mathrm{Eu}$ provides the closest match to the actual Solar age, while in general - like for $\mathrm{Th} / \mathrm{Hf}$ - there it the tendency to grossly overestimate the age. The photospheric solar abundance of europium provided by Lawler et al. (2007) is exactly the same we are using. Obviously, the complex chemical history of the Sun together with uncertainties in the production ratios make it very difficult to obtain a reliable Solar age by radioactive dating.

In this context it is perhaps interesting to note that Sneden et al. (2009) find that the solar photospheric Hf abundance is deviating from the meteoritic value in contrast to all its preceeding (in atomic number) rare earth elements. Similar, the solar $r$-process abundance pattern corresponds to the one of $r$-process enriched iron-poor halo stars except for Hf which appears relatively depleted in the Sun. Of course, a higher $r$-process abundance of Hf in the Sun would even lead to a greater solar age aggravating the problem in the radioactive dating.

\section{Conclusions and remarks}

The accuracy of radioactive cosmo-chronometry is not going to be extremely high on a single star. A realistic estimate of what future instrumentation will be able to achieve does likely not allow one to hope for a better than $2 \mathrm{Gyr}$ precision, taking into account observational uncertainties only, leaving aside the systematics associated to production rates and chemical enrichment models. Although high-mass $n$-capture elements are currently believed to be produced in a fairly well understood manner by the so-called "main" $r$-process (Farouqi et al. 2008; Kratz et al. 2007), the quantitative determination of the production ratios $\beta$ between the stable and the unstable elements still bear significant uncertainties.

Since in photon noise dominated spectra the measurement error on the equivalent width decreases linearly with the observational $\mathrm{S} / \mathrm{N}$ ratio, the attainable dating precision increases roughly linearly with the $\mathrm{S} / \mathrm{N}$ ratio. This constitutes a major practical challenge to the application of the method, since many of the involved lines are in the blue part of the optical spectrum (e.g. for Th, U, and Hf), and the natural target of the analysis are cool stars.

If possible, the decaying element should be chosen such that its mean lifetime $\tau$ is similar to the age one wants to measure. This applies also to the "effective" $\tau$ of a chronometric pair constituted by two decaying species. From this perspective, the pairs $\mathrm{U} / \mathrm{Th}, \mathrm{Th} /$ stable, and U/stable are well suited to measure ages of old objects around $10 \mathrm{Gyr}$ of age. No other naturally occurring isotope has a mean lifetime providing a similarly close match.

Potentially important systematic spectroscopy-related effects that we feel deserve further study to fully exploit the potentials of radioactive dating are effects related to departures from thermodynamic equilibrium and gas-dynamics on the line formation of the chronometric species. For instance, the application of a $3 \mathrm{D}$ radiation-hydrodynamical model atmosphere by Caffau et al. (2008) led to a downward revision of the photospheric Solar Th abundance by 0.1 dex. This corresponds to a decrease of Th ages by 4.7 Gyr.

Globular clusters provide fairly large samples of metal-poor, bright, coeval and chemically homogeneous stars. Simultaneous measurement of abundances in these stars allows us to reduce the observational, statistical uncertainties. This could permit to derive $r$-process production ratios empirically by calibration against the known "photometric" age. To our knowledge, this has never been attempted so far.

Acknowledgements. The authors H.-G.L., P.B, and L.S. acknowledge financial support from EU contract MEXT-CT-2004-014265 (CIFIST).

\section{References}

Arlandini, C., Käppeler, F., Wisshak, K., et al. 1999, ApJ, 525, 886 Arnould, M., Goriely, S., \& Takahashi, K. 2007, Phys. Rep., 450, 97 Caffau, E., Sbordone, L., Ludwig, H.-G., et al. 2008, A\&A, 483, 591 Cayrel, R. 1988, The Impact of Very High S/N Spectroscopy on Stellar Physics, IAU Symp., 132, 345

Cowan, J. J., Thielemann, F.-K., \& Truran, J. W. 1991, ARA\&A, 29, 447

Cowan, J. J., Pfeiffer, B., Kratz, K.-L., et al. 1999, ApJ, 521, 194

Cowan, J. J., Sneden, C., Burles, S., et al. 2002, ApJ, 572, 861

del Peloso, E. F., da Silva, L., \& Porto de Mello, G. F. 2005a, A\&A, 434, 275

del Peloso, E. F., da Silva, L., \& Arany-Prado, L. I. 2005b, A\&A, 434, 301

del Peloso, E. F., da Silva, L., Porto de Mello, G. F., \& Arany-Prado, L. I. 2005c, A\&A, 440, 1153

Farouqi, K., Kratz, K.-L., Cowan, J. J., et al. 2008, Proceedings of First Stars III conference, Santa Fe, New Mexico, 15-20 July 2007, ed. B. W. O’Shea, A. Heger, \& T. Abel, 309

Frebel, A., Christlieb, N., Norris, J. E., et al. 2007, ApJ, 660, L117

Goriely, S., \& Clerbaux, B. 1999, A\&A, 346, 798

Grevesse, N., \& Sauval, A. J. 1998, Space Sci. Rev., 85, 161

Hill, V., Plez, B., Cayrel, R., et al. 2002, A\&A, 387, 560

Honda S., Aoki W., Kajino T., et al. 2004, ApJ, 607, 474

Ivans, I. I., Simmerer, J., Sneden, C., et al. 2006, ApJ, 645, 613

Johnson, J. A. 2002, ApJS, 139, 219

Johnson, J. A., \& Bolte, M. 2001, ApJ, 554, 888

Kratz, K.-L., Farouqi, K., Pfeiffer, B., et al. 2007, ApJ, 662, 39

Lawler, J. E., Hartog, E. A. D., Labby, Z. E., et al. 2007, ApJS, 169, 120

Lawler J. E., Sneden C., Cowan J. J., Ivans I. I., \& Den Hartog E. A., 2009, ApJS, 182, 51

McWilliam, A., Preston, G. W., Sneden, C., \& Searle, L. 1995, AJ, 109, 2757

Mucciarelli, A., Caffau, E., Freytag, B., Ludwig, H.-G., \& Bonifacio P. 2008, A\&A, 484, 841

Roederer, I. U., Kratz, K.-L., Frebel, A., et al. 2009, ApJ, 698, 1963

Schatz, H., Toenjes, R., Pfeiffer, B., et al. 2002, ApJ, 579, 626

Simmerer, J., Sneden, C., Cowan, J. J., et al. 2004, ApJ, 617, 1091

Sneden, C., Cowan, J. J., Ivans, I. I., et al. 2000a, ApJ, 533, L139

Sneden, C., Johnson, J., Kraft, R. P., et al. 2000b, ApJ, 536, L85

Sneden, C., Cowan, J. J., Lawler, J. E., et al. 2003, ApJ, 591, 936

Sneden, C., Cowan, J. J., \& Gallino, R. 2008, ARA\&A, 46, 241

Sneden C., Lawler J. E., Cowan J. J., Ivans I. I., \& Den Hartog E. A., 2009, ApJS, 182, 80

Spergel, D. N., Bean, R., Doré, O., et al. 2007, ApJS, 170, 377

Vandenberg, D. A., Stetson, P. B., \& Bolte, M. 1996, ARA\&A, 34, 461

Yong D., Karakas A. I., Lambert D. L., Chieffi A., \& Limongi M., 2008, ApJ, 689, 1031

Westin, J., Sneden, C., Gustafsson, B., \& Cowan, J. J. 2000, ApJ, 530, 783 
Table A.1. $r$-process elemental abundances in metal-poor stars and the Sun for radioactive dating.

\begin{tabular}{|c|c|c|c|c|c|c|c|c|}
\hline Object & Reference & {$[\mathrm{Fe} / \mathrm{H}]$} & $A(\mathrm{Eu})$ & $A(\mathrm{Hf})$ & $A(\mathrm{Os})$ & $A(\mathrm{Ir})$ & $A(\mathrm{Th})$ & $A(\mathrm{U})$ \\
\hline HD 115444 & Cowan et al. (1999) & -2.7 & -1.5 & & & & -2.1 & \\
\hline HD 115444 & Westin et al. (2000) & -2.9 & $-1.63 \pm 0.07$ & & $-0.55 \pm 0.11$ & & $-2.23 \pm 0.11$ & $<-2.6$ \\
\hline CS 22892-052 & Cowan et al. (1999) & -3.1 & -0.9 & & & & -1.6 & \\
\hline CS 22892-052 & Sneden et al. $(2000 \mathrm{a})^{a}$ & -3.1 & $-0.89 \pm 0.12$ & $-0.90 \pm 0.14$ & $-0.10 \pm 0.38$ & & $-1.55 \pm 0.08$ & \\
\hline CS 22892-052 & Sneden et al. (2000a) & -3.1 & $-0.93 \pm 0.09$ & & $-0.05 \pm 0.10$ & $+0.00 \pm 0.26$ & $-1.60 \pm 0.07$ & \\
\hline CS 22892-052 & Sneden et al. (2003) & -3.1 & $-0.95 \pm 0.03$ & $-0.98 \pm 0.10$ & $+0.02 \pm 0.13$ & $-0.10 \pm 0.10$ & $-1.57 \pm 0.10$ & $<-2.3$ \\
\hline $\mathrm{BD}+173248$ & Cowan et al. (2002) & -2.0 & $-0.67 \pm 0.05$ & & $+0.45 \pm 0.10$ & $+0.30 \pm 0.13$ & $-1.18 \pm 0.10$ & $-2.0: \pm 0.30$ \\
\hline HD 221170 & Ivans et al. (2006) & -2.18 & $-0.86 \pm 0.07$ & $-0.94 \pm 0.04$ & $+0.16 \pm 0.10$ & $+0.02 \pm 0.13$ & $-1.46 \pm 0.05$ & \\
\hline CS 31082-001 & Hill et al. (2002) & -2.90 & $-0.63 \pm 0.04$ & $-0.59 \pm 0.17$ & $+0.43 \pm 0.12$ & $+0.20 \pm 0.09$ & $-0.98 \pm 0.02$ & $-1.92 \pm 0.11$ \\
\hline M51 K341 & Sneden et al. (2000b) & -2.32 & $-0.88 \pm 0.09$ & & & & $-1.47 \pm 0.10$ & \\
\hline M51 K462 & Sneden et al. (2000b) & -2.25 & $-0.61 \pm 0.09$ & & & & $-1.26 \pm 0.10$ & \\
\hline M51 K583 & Sneden et al. (2000b) & -2.34 & $-1.22 \pm 0.09$ & & & & $-1.7: \pm 0.10$ & \\
\hline M4 L1411 & Yong et al. (2008) & -1.23 & & +0.08 & & & -0.68 & \\
\hline M4 L1501 & Yong et al. (2008) & -1.29 & & -0.02 & & & -0.63 & \\
\hline M4 L1514 & Yong et al. (2008) & -1.22 & & +0.03 & & & -0.83 & \\
\hline M4 L2406 & Yong et al. (2008) & -1.30 & & -0.12 & & & -0.88 & \\
\hline M4 L2617 & Yong et al. (2008) & -1.20 & & +0.08 & & & -0.48 & \\
\hline M4 L3209 & Yong et al. (2008) & -1.25 & & +0.03 & & & -0.68 & \\
\hline M4 L3413 & Yong et al. (2008) & -1.23 & & -0.02 & & & -0.58 & \\
\hline M4 L4511 & Yong et al. (2008) & -1.22 & & +0.03 & & & -0.63 & \\
\hline M4 L4611 & Yong et al. (2008) & -1.09 & & +0.18 & & & -0.68 & \\
\hline M5 IV-81 & Yong et al. (2008) & -1.28 & & -0.12 & & & -0.58 & \\
\hline M5 IV-82 & Yong et al. (2008) & -1.33 & & -0.22 & & & -0.68 & \\
\hline HD 108577 & Johnson \& Bolte (2001) & -2.38 & $-1.48 \pm 0.02$ & & & & $-1.99 \pm 0.07$ & \\
\hline HD 115444 & Johnson \& Bolte (2001) & -3.15 & $-1.81 \pm 0.03$ & & & & $-2.36 \pm 0.07$ & \\
\hline HD 186478 & Johnson \& Bolte (2001) & -2.61 & $-1.56 \pm 0.06$ & & & & $-2.26 \pm 0.07$ & \\
\hline $\mathrm{BD}+82856$ & Johnson \& Bolte (2001) & -2.12 & $-1.16 \pm 0.06$ & & & & $-1.66 \pm 0.10$ & \\
\hline M92VII-18 & Johnson \& Bolte (2001) & -2.29 & $-1.48 \pm 0.09$ & & & & $-1.97 \pm 0.07$ & \\
\hline Sun & Grevesse \& Sauval (1998) & +0.0 & & & $+1.45 \pm 0.10$ & $+1.35 \pm 0.10$ & & \\
\hline Sun & Mucciarelli et al. (2008) & +0.0 & $+0.52 \pm 0.03$ & & & & & \\
\hline Sun & Caffau et al. (2008) & +0.0 & & $+0.87 \pm 0.04$ & & & $+0.08 \pm 0.03$ & \\
\hline
\end{tabular}

${ }^{a}$ Sneden et al. analysed two different spectra for CS 22892-052 providing two independent measurements. For this reason the object/reference combination is listed twice.

Table A.2. $r$-process production ratios.

\begin{tabular}{lccccc}
\hline \hline Reference & \multicolumn{5}{c}{ Production ratio $\beta$} \\
& Th/Eu & Th/Hf & Th/Os & Th/Ir & U/Th \\
\hline Kratz et al. (2007) & 0.453 & 0.862 & 0.093 & 0.089 & 0.638 \\
Sneden et al. (2003) & 0.420 & & & & \\
Schatz et al. (2002) & 0.468 & & 0.071 & 0.066 & \\
Cowan et al. (2002) & 0.507 & & & 0.087 & \\
\hline
\end{tabular}

\section{Appendix A: Tables of elemental abundances, production ratios, and solar $r$-process fractions}

The following tables list the abundances, production ratios, and solar system $r$-process fractions that we compiled from the literature for the purpose of radioactive dating. In Table A.1,
Table A.3. Solar $r$-process fractions $f_{\mathrm{r}}$ for various elements $X$.

\begin{tabular}{lll}
\hline \hline$X$ & $f_{\mathrm{r}}$ & Reference \\
\hline $\mathrm{Eu}$ & 0.942 & Arlandini et al. (1999) \\
$\mathrm{Hf}$ & 0.566 & Lawler et al. (2007) \\
$\mathrm{Os}$ & 0.92 & Simmerer et al. (2004) \\
$\mathrm{Ir}$ & 0.99 & Simmerer et al. (2004) \\
\hline
\end{tabular}

exclusively spectroscopically determined (as opposed to meteoritic) abundances are given for the Sun. In Table A.2 of production ratios, the values for Kratz et al. (2007) refer to the ones listed as "Fe-Seed fit 2 " in Table 2 of their paper. Kratz and collaborators report three different estimates of the various production ratios. 